A Pancultural Perspective on the Fading Affect Bias in Autobiographical Memory

Timothy D. Ritchie

University of Limerick

\author{
Tamzin J. Batteson \\ Rosalind Franklin \\ University \\ Georgie V. Ferguson \\ University of Montana
}

\author{
Annette Bohn \\ Aarhus University \\ Robert W. Schrauf \\ Pennsylvania State University \\ W. Richard Walker \\ Winston-Salem State University
}

Matthew T. Crawford Victoria University of Wellington

Rodney J. Vogl Christian Brothers University

This is an Author's Accepted Manuscript of an article published in Memory, epub ahead of print published online:14th February 2014, available online

at: http://www.tandfonline.com/10.1080/09658211.2014.884138

Changes resulting from the publishing process, such as peer review, editing, corrections, structural formatting, and other quality control mechanisms may not be reflected in this document. Changes may have been made to this work since it was submitted for publication. 


\section{Authors' Notes}

We thank the following individuals for their help with data collection, entry and preparation for analysis: Sarah Cadogan, Anahera Gildea, Shawnda Marsh, Kirsty Maya Roopun, and Rosalyn Shelton. 


\begin{abstract}
The Fading Affect Bias (FAB) refers to the negative affect associated with autobiographical events fading faster than the positive affect associated with such events, a reliable and valid valence effect established by researchers in the U.S.A. The present study examined the idea that the $\mathrm{FAB}$ is a ubiquitous emotion regulating phenomenon in autobiographical memory that is present in people from a variety of cultures. We tested for evidence of the FAB by sampling more than 2,400 autobiographical event descriptions from 562 participants in 10 cultures around the world. Using variations on a common method, each sample evidenced a FAB: Positive affect faded slower than negative affect did. Results suggest that in tandem with local norms and customs, the FAB may foster recovery from negative life events and promote the retention of the positive emotions, within and outside of the U.S.A. We discuss these findings in the context of Keltner and Haidt's (1999) levels of analysis theory of emotion and culture.
\end{abstract}


A Pancultural Perspective on the Fading Affect Bias in Autobiographical Memory The human experience is replete with personal, emotional events. The emotions associated with life events can be positive or negative, fleeting or long lasting, disruptive or soothing. Emotions are constructed, in part, by biological and social mechanisms that moderate the affective experience and its expression. Much of the research on emotions has focused on immediate consequences for thought and behavior (Barrett, Niedenthal, \& Winkielman, 2005; Damasio, 2003; Forgas, 2000). However, a growing body of research has begun to examine the fate of personal events' emotions over time (Walker \& Skowronski, 2009). This research suggests that many emotions evidence an overall pattern of affective fading. For many events, their associated emotions become less intense with the passage of time, some gradually, others abruptly (Gibbons, Lee, \& Walker, 2011; Ritchie \& Batteson, 2013). Importantly, this tendency for affective fading is biased such that negative emotions, on average, fade significantly faster than do positive emotions. This pattern of affect change is the Fading Affect Bias (FAB).

The evidence for such a differential fading of positive and negative affect is robust and shows demonstratively that many adults evidence a FAB. Nonetheless, the majority of the early research on the FAB involved North American samples of university students, particularly those from the United States (Walker, Vogl, \& Thompson, 1997). There remains the possibility that the FAB represents a cultural phenomenon, such that it may only be present in the relatively homogeneous samples thus far examined, within the U.S. To our knowledge, no prior work has provided data that enable us to compare the FAB cross-culturally' thus, the possibility of a culture-specific effect remains open. For example, ethnic groups in the U.S., such as AfricanAmericans and Native American tribes have each historically been the target of systematic abuse 
and discrimination. Such prejudice and discrimination may have affected how members of these groups think about and express their positive and negative emotions, especially feelings

prompted by reminiscing past events (Clark, Anderson, Clark, \& Williams, 1999; Dorgan, 2010).

We think that the differential fading of negative and positive affect represents a general affect regulation mechanism that is not constrained by cultural or linguistic norms. The goal of the current research is to review the evidence of the FAB across cultures, and to reveal the extent to which this pattern of affective change is not tethered to U.S. cultures. To achieve this goal, we pooled 10 samples of autobiographical events from individuals from around the world to assess their perceived changes in the affect associated with their own personal events. A FAB in all 10 samples would bolster the notion that it may indeed be a pancultural phenomenon, similar to how facial expressions occur universally (Ekman \& Friesen, 1971; Elfenbein \& Ambady, 2002).

To examine the FAB across cultures we compiled relevant data sets from several research teams. Even if the FAB occurs in each of the ten samples, it would not necessarily suggest that such emotional changes were equivalent. Nonetheless, if the FAB occurs across the diverse samples in the present manuscript, it could help reveal emotion regulation patterns, their adaptive neural structures, and related cognitive positivity biases. We can tentatively dismiss the notion that cultural and social norms underpin this valence effect.

\section{The Fading Affect Bias}

The initial research on phenomena related to the FAB began over 80 years ago. Meltzer (1930) examined the retention of positive and negative memories in which participants recorded events that had occurred during a recent holiday break. His participants indicated whether each event was pleasant or unpleasant. Six weeks later, they recalled as many of their recorded events 
as possible. They recalled more positive than negative events during the recall session. Meltzer (1931) replicated the results of the earlier study and interpreted the biased recall as a sign of personal adjustment. In a similar study, Watters and Leeper (1936) asked participants to recall as many positive and negative events as they could that had happened to them over a recent holiday season. The participants then rated the affect of each event on a scale that ranged from -3 (extremely unpleasant) to +3 (extremely pleasant). Participants returned for a second session that was for some participants as long as 4.5 months after the original session, and were asked to recall as many events from the first session as possible. The participants recalled more positive than negative events, a result that was again interpreted as positive coping.

Early research was not limited to the relative proportion of recalled positive and negative events. At least one study examined the extent to which positive emotions and negative emotions changed over time. For instance, Cason (1932) asked participants to each describe three to eight emotional memories from the previous week, and then to rate how they felt about each memory via an 11-point scale. They made similar judgments three weeks after recording the events. Both judgments attempted to record the emotionality associated with each event. Cason revealed that the feelings associated with all events became weaker over time, and importantly, the fading was larger for negative events than for positive events.

Holmes (1970) conducted a diary study to examine the relation of emotional intensity to the fading affect over time. He found that the affect for unpleasant events faded more than the affect for pleasant events. Nearly three decades later, Walker et al. (1997) examined the fading of emotions over time, including a group for a three-month retention interval (43 participants), a group for a one-year interval (6 participants), and one participant at a 4.5-year interval. By 
recording events on the day that the events occurred, the researchers ensured that the data would not be confounded by an event selection bias. The results supported the hypothesis that the unpleasant emotions associated with events faded more rapidly over time than pleasant emotions associated with events, and this differential fading increased across long retention intervals.

Gibbons, Lee, and Walker (2011) reported evidence of how rapidly the differential fading of pleasant and unpleasant emotions occurs. They found that the FAB occurred within 24 hours after an event and that it persisted for at least three months. Recently, Ritchie and Batteson (2013) conducted a study that involved six separate assessments of multiple day-old events per person across a two-week period. In all, these results converged with the extant FAB research by demonstrating the speed and ease with which the FAB occurs, as well as its persistence.

These studies suggest that the FAB occurred via a variety of research methods, including diary and retrospective procedures, ratings taken in a single session and repeated measures; thus far, such studies produced consistent results. The present research relied on two distinct approaches to studying the FAB (i.e., contemporaneous affect ratings and test-retest affect ratings); we report the method details later. FAB research spans at least 80 years, primarily among U.S. samples. The consistent pattern of findings suggests that the FAB may help individuals to regulate their emotions, overcome past negative events, and to maintain a positive self-concept (Ritchie, Skowronski, Cadogan, \& Sedikides, in press). Indeed, many participants' event descriptions and affect ratings suggest that they frequently overcame adversity and savored good times. Oftentimes, reminiscence fosters the narration of a positive life story. Such narratives emphasize the ability of individuals to reframe their former misery or failure and to recover from such events, to ultimately achieve personal resolve and growth (McAdams, 2013). 
Under the circumstances that have been examined empirically, the FAB suggests that pleasant affect from the past remains strong to a person in the present. Accessibility of positive information from the past may contribute to a range of positivity biases that have been documented by researchers (Ehrenreich, 2009; Twenge \& Campbell, 2009). We next focus on the central question of the present study: Is the FAB a predominantly U.S. phenomenon?

\section{The FAB Panculturally: Ubiquitous Emotion Regulation?}

It is true that up to now most of the published research on the FAB was conducted in the U.S. (Walker \& Skowronski, 2009). It could be that across cultures, different patterns of emotional change in autobiographical event recall will occur (i.e., not a FAB). We think that it is unlikely that FAB is a phenomenon of the U.S.; however, it is an open empirical issue that we address in the present research. As mentioned earlier, we view the FAB as part of emotion regulation. Indeed, related to emotion regulation strategies, the FAB may result from a deliberate and effortful engagement with and reappraisal of one's own past events and their associated feelings. The FAB also occurs automatically, often requiring little or no effort, and typically occurs beneath a person's own awareness. A deliberate approach to the FAB requires memorial effort. For instance, a person reminisces on the pleasant memories and feelings that are associated with a recent vacation, or perhaps dwells on the flight delay and expensive car rental (Joorman \& Siemer, 2004). The former reinforces the pleasant feelings associated with the event, whereas the latter may weaken the positive feelings associated with the event. To date, relatively little research on emotion regulation has considered the emotional information that a person retrieves from their own memory. For instance, Joorman and Siemer (2004) reported evidence from two studies suggesting that, while in a bad mood, nondysphoric individuals (versus their 
mildly depressed counterparts) engage in mood-regulatory processes by sometimes accessing positive autobiographical memories.

Emotion regulation concerns both internal resources (i.e., self-protection, selfenhancement) and external resources (i.e., social support, norms); individuals rely on each to deal with their own emotions in a manner that is acceptable to their immediate environment (Koole, 2009). We think that the FAB is a predominantly automatic, internal process governed, in part, by the same neural systems involved with the experience of mental time travel and the experience of event-related emotions in the present (Piefke et al., 2004). Comparatively less is known about the extent to which such mood-regulating phenomena occur across cultures.

Consistent with pancultural emotion regulation ideas reviewed by De Leersnyder, Boiger, and Mesquita (2013), one culture might encourage the elaboration of emotional experiences whereas another might dissuade such elaboration, leading members of one culture to respond to a situation with excitement, contentment or joy, while members of another culture might respond with fear, embarrassment or shame (Scherer \& Brosch, 2009). Moreover, cultures have rules for how emotions should be displayed or conveyed to others (Matsumoto \& Hwang, 2010). For example, women in many Western cultures are encouraged to smile whereas women in some Eastern cultures often cover a smile, as it represents a violation of a culture-specific display rule (La France, Hecht, \& Paluck, 2003). De Leersnyder et al.'s (2013) review emphasizes that emotion regulation across cultures goes well beyond emotion display rules. Cultural emotion regulation extends to cognitive appraisal processes, such as affect appraisals that a person associates with their daily life events. 
Despite the plausible counter-hypothesis to the FAB (i.e., the FAB is not a pancultural phenomenon), we think that the affect prompted by the recall of personal events occurs in tandem with a compendium of emotion regulation strategies, the deliberate and effortful as well as the implicit and spontaneous. Within the context of emotion regulation we view the FAB as a robust intrapersonal process, indicative of emotion and self-regulation. For instance, the FAB is weak or not present among individuals with symptoms of depression, anxiety, an eating disorder, or excessive narcissism (Ritchie \& Dromey, 2014; Ritchie, Walker, Marsh, \& Skowronski, 2014; Walker, 1998; Walker et al., 2003a; Walker, Yancu, Skowronski, \& Vogl, 2014).

Theorists posit that culture modulates but does not entirely determine a person's emotional experience. For example, the Neuro-Cultural Theory of Emotion (Ekman, 1972) was one of the first theories to attempt to reconcile the innateness of emotion with the apparent effects of culture on emotion. This theory suggested that an emotional event or memory would initially trigger a universal set of responses in a person that would then be filtered by the display rules of the person's culture. The display rules would intensify, neutralize, or mask the emotional experience and expression of the individual.

Research by Keltner and Haidt (1999) supported this theory and expanded on it by examining its social functions, namely to 1 ) help a person survive and reproduce (individual level), 2) help two individuals form a relationship bond (dyadic level), 3) negotiate social roles within a group (group level), and 4) impart specific societal values and customs (cultural level). They suggested that the functions of emotions at the first two levels, individual and dyadic, are universal for the experience and expression of emotion, whereas the functions of emotions at the 
third and fourth levels, group and culture, allow for much more variation in emotional experience and expression (Keltner, Haidt, \& Shiota, 2006).

According to Keltner and Haidt's (1999) Levels of Analysis theory of emotion, the FAB could be the result of cultural norms and values. People from different backgrounds and countries could experience different changes in the affect associated with their own event memories. Given that most of the work demonstrating the FAB was collected within the U.S., the FAB could reflect the common U.S. attitude to look on 'the bright side' and to approach obstacles with a 'can-do' attitude. The prediction suggests that samples of U.S. citizens (AfricanAmericans, Caucasians, Native Americans, etc.) would show a stronger FAB than samples from people from other parts of the world (Africa, Europe, New Zealand, etc.). Does a person's worldview imbue their personal life story and narrative with an exaggerated sense of positivity, such that individuals from cultures who do not share such a worldview do not evidence such heightened positivity in their life stories? We do not think so; however, it is possible.

In terms of Keltner and Haidt's (1999) model, we think that the FAB is a universal memorial process that fosters the minimization of negative affect and retention of positive affect of event memories, and that such processes operate independently of local customs and norms. We argue that the FAB is likely not a uniquely U.S. phenomenon; rather, it is adaptive and part of a set of processes that foster emotion regulation. Thus, from this perspective we predicted the FAB would occur not only among samples across the U.S. but also among samples across the globe. 


\section{The Present Study}

The goal of the present research was to demonstrate evidence of the FAB in samples of participants from various cultures around the world. The present study examined data from 10 different samples collected by researchers at six universities with access to different ethnic samples. These samples include African-Americans, Ghanaians, Black British, Caucasian Americans, Caucasian British, Germans, Irish, Native American, New Zealanders of European descent, and New Zealanders of Maori and Pasifica descent. Further, we reanalyze data from Puerto Ricans in the context of the FAB. Data from two samples were previously published (Bohn \& Berntsen, 2007; Schrauf \& Hoffman, 2007); the remaining samples were previously unpublished.

\section{Method}

Participants. Table 1 includes the participant sample sizes and the autobiographical event sample sizes for each study. Sample 1 included 58 African-American citizens (41 women, 17 men) who were undergraduate students at Winston-Salem State University, a historically African-American university. They were between the ages of 18 and 51 years $(M=20.60, S D=$ 4.58). Sample 2 included 14 Ghanaian citizens ( 11 women, 2 men, 1 unidentified) whose ages ranged from 18 to 30 years $(M=22.74, S D=4.44)$. All were fluent in English; 13 were students at the University of Cape Coast. Sample 3 included 30 Caucasian American citizens (16 women, 14 men) from Kansas State University whose ages ranged from 18 to 25 years $(M=19.52, S D=$ 1.87). These data were from an unpublished portion of a doctoral dissertation (Walker, 1998). Next, Sample 4 included American citizens (13 women, 11 men, 1 unidentified) from various tribes in the United States (i.e., the Alabama Coushatta, Cherokee, Choctaw, Creek, Houma, 
Shoshone, and Seminole tribes). All self-identified as Native American or American Indian, and were between the ages of 18 and 65. Sample 5 included 180 German citizens (86 women, 93 men) between the ages of 29 and 82 years $(M=54.41, S D=14.07)$. They were at least 16 years old in 1989, when the Berlin Wall fell. Bohn and Berntsen (2008) reported additional details. Sample 6 included 43 Caucasian citizens of New Zealand (37 women, 6 men), who were undergraduate students at Victoria University of Wellington. Sample 7 included 16 Maori and Pasifica citizens of New Zealand (14 women, 2 men), who were undergraduate student at Victoria University of Wellington (age information not recorded). Sample 8 included 61 mostly Caucasian British (90\%) undergraduate students $(n=22)$ at the University of Southampton (UK); adults from the Southampton community $(n=21)$; and, older adults from a residential nursing home in Hampshire County $(n=18)$. They included 44 women, 14 men, and three participants who did not specify their gender, between the ages of 18 and 99 years $(M=48.14$, $S D=26.78)$. Sample 9 included 100 Caucasian Irish citizens (57 women, 43 men) between the ages of 18 and 66 years $(M=28.33, S D=12.46)$ from the University of Limerick and the nearby community in County Limerick. Finally, Sample 10 included 33 Black British citizens (28 women, 5 men) between the ages of 18 and 39 years $(M=21.23, S D=4.27)$, enrolled in a psychology course at Middlesex University in London.

Procedure. Across all samples, each participant reviewed and signed (except those in Sample 5) an Informed Consent prior to taking part in the study. Each study’s design and procedure complied with the university’s Institutional Review Board, in accordance with standards of research ethics and code of conduct (American Psychological Association, 2010; British Psychological Society, 2009). Across all studies the research procedure was the same: 
participants were prompted to recall one or more autobiographical events from their own lives, and then rate each event via scales of affect pleasantness. Affect scales are described below for each study. In return for their time and contribution to each study, most participants received credit toward their course grade (Studies 1, 3, 6, 7), received \$5,USD (Study 2), offered chocolate (Study 9) or offered a chance to win a gift certificate (Study 8, 10).

In Samples 1, 2, 6, and 7, participants had 20 min to recall 10 autobiographical events from their own lives, five positive events and five negative events. They were asked to describe each event in sufficient detail (e.g., time, location, sensory information). After the recall period, participants were asked to rate two indicators of affect for each event. For Sample 2, in 2010 an undergraduate student in a study abroad program at the University of Cape Coast collected these data. In Sample 3, participants had 20 min to recall six autobiographical events from their own lives. They were instructed to recall both positive and negative events and were encouraged to recall as many events of each type that they wanted. In Sample 4, participants had 15 min to recall four autobiographical events from their own lives. They were instructed to recall two positive events and two negative events and to describe each event in sufficient detail. An undergraduate research assistant of Native American descent guided their session in a semiprivate venue, while attending a week-long cultural event held on a reservation in the southeast region of the United States.

In Sample 5, participants each responded to a questionnaire about a specific personal event, namely their own recollection of the fall of the Berlin Wall. In that sample, participants were members of the conservative Christlich Demokratische Union party (who likely viewed the fall of the Berlin Wall positively), the Partei des Demokratischen Sozialismus (who likely 
viewed the fall of the Berlin Wall negatively), or unaffiliated with either party. The questionnaire included items for how each participant had felt about the event when it happened, and how they felt about the event now. In Sample 6, participants had 20 min to recall 10 autobiographical events from their own lives, five positive events and event negative events. They were asked to describe each event in sufficient detail (e.g., time, location, sensory information). After the recall period, participants were asked to rate two indicators of affect for each event.

In Sample 8, participants recalled and described two positive events and two negative events [“an event that you felt positively (negatively) about at that time”]. One of each was from five years ago, and one from 10 years ago. The order in which this valence manipulation occurred was counterbalanced: Half of participants recalled positive events first, and half recalled negative events first. In Sample 9, a research assistant asked participants to each recall four important or four unimportant autobiographical events that were either affectively positive or negative.

In sample 10, the course instructor e-mailed participants an informed consent form, instructions that prompted them to recall event memories, and a spreadsheet. They recorded in the spreadsheet a description of an event's mental image for two pleasant events or two unpleasant events that had occurred the day before receiving the e-mail.

Event Affect Ratings and Affect Change. In Samples 1-7, the participants each rated the affect associated with an event at its occurrence, Initial Affect. They also rated the affect that was prompted when an event was recalled, Current Affect. Ratings for each event on both scales included a 7-point, bipolar scale that ranged from -3 (extremely unpleasant) to 0 (neutral) to +3 (extremely pleasant). 
In Samples 8-9, the participants each rated the Initial Affect for each of their events via the item: “When it happened, how did the event make you feel?” Participants rated each event's Current Affect via the following item: "When you remember the event now, how does remembering the event make you feel?” Both items used the same response scale, anchored according to valence. For negative items, the response scale ranged from 1 (slightly unpleasant) to 6 (exceptionally unpleasant). For positive events, the response scale ranged from 1 (slightly pleasant) to 6 (exceptionally pleasant).

Finally, in Sample 10, the participants each rated the Initial Affect for each of their events on a 6-point scale that ranged from 1 (pleasant or unpleasant) to 6 (very pleasant or very unpleasant). Two weeks later, participants received their event image descriptions via email. Without reviewing their initial affect ratings, they rated each event via the same scales described above.

\section{Results}

Analytic Strategy. In all but one of the samples that we report (Sample 5, Germany) we prompted multiple autobiographical events from each participant; hence, most of the data were clustered, autobiographical events within persons. For nine out of 10 analyses, the data behooved us to consider variance in affect fading that could be attributable to individual differences in event selection. In data with more than one level, the residuals among each person's event cluster may correlate with each other, due in part to systematic individual differences. That is, in some samples, some participants could have selected all affectively potent events from their past, whereas other participants in the same sample could have selected events consisting of a variety of affective potency. While we did not expect such individual differences to exert systematic 
influence on the FAB across all studies, such differences were possible and manageable. In our main analyses, we treated each cluster of events as a person via a dummy-coded variable; however, we did not treat this variable as its own level in our data.

For each relevant sample, we first accounted for the variance explained in affect fading by each person. In Table 3, we refer to this level effect as “Person”. The $R^{2}$ from this model acted as a baseline against which we compared a second model's $R^{2}$ that included both Person and "Event Valence.” Thus, we report the increase in $R^{2}$ that was attributable to Event Valence, while considering the potential individual differences in event selection. For analysis, we used IBM SPSS 20 for Windows, specifically the General Linear Model algorithm and its Least Squared Means, and Model $R^{2}$ for a common metric of effect size across samples. Next, as noted earlier Sample 5 (Germany) included one event per person; hence, we needed no person dummy variable in the model. Finally, we assessed the participants in Sample 10 (Black British, UK) on two occasions, two weeks apart; hence, for analysis we employed the Repeated Measures GLM in IBM SPSS 20.

Descriptive Statistics. Table 1 illustrates our samples by country and culture, the sample size of participants and the number of rated events obtained in each sample; across studies, the latter acted as the primary unit of analysis. Table 2 includes the absolute values of the means and the standard errors for initial affect and current affect ratings, and the mean affect fading score per event valence, not controlling for potential individual differences in event recall.

The Fading Affect Bias. We first report the findings of the relation between event valence and affect fading, not controlling for the potential influence of individual differences in event selection. We then report the results for any models that were influenced substantially by 
such individual differences. In all, the results below reflect the statistical differences between the means reported in Table 2 and the incremental variance accounted for by event valence, relative to individual differences in events selection, as reported in Table 3.

Consistent with prior research and our hypothesis, the FAB valence effect occurred in each of the samples in the present manuscript. On average, positive personal events retained their affective pleasantness longer than negative personal events retained their affective unpleasantness. Table 3 includes the effect sizes for each analysis. The statistics for the valence effect in each model are as follows: 1) African-American, $F(1,570)=250.01, p<.0005 ; 2)$ Ghanaian, $F(1,119)=57.84, p<.0005 ; 3)$ Caucasian, USA, $F(1,184)=109.52, p<.0005 ; 4)$ Native American, $F(1,83)=38.26, p<.0005$; 5) German, $F(1,178)=84.17, p<.0005$; 6) New Zealander European, $F(1,427)=169.39, p<.0005 ; 7)$ New Zealander Maori \& Pasifica, $F(1$, 159) $=28.56, p<.0005 ; 8)$ Caucasian British, $F(1,236)=33.18, p<.0005 ; 9)$ Irish, $F(1,391)=$ 102.66, $p<.0005$; and, 10) Black British, UK, $F(1,63)=7.77, p=.007$.

Next, the valence effect remained statistically significant with the individual differences in event selection in the model, for all but the Irish sample. In this sample, variance in affect fading was better explained by individual differences in event selection than event valence, such that the valence effect was rendered non-significant, $F(1,292)=0.00, p=1.0$.

Finally, we examined whether the presence of FAB could be explained by participant age. Valence effects can be significantly influenced by participant age, such that older individuals tend to show a positivity bias (e.g., Charles \& Carstensen, 2010). For the seven of 10 samples that recorded participants' age, inclusion of age into the model exerted no influence on the FAB. In six of those seven samples, age positively predicted variance in fading. Despite the 
statistical relation between person age and event affect fading, age by itself did not account for the FAB. Results from each of these additional analyses are available upon request.

Attempt to Replicate via Puerto Rican Sample. Schrauf and Hoffman (2007) examined personal event affect using a method that could substantiate evidence supporting the FAB. However, their method did not enable a straightforward comparison of results to the other samples in the present manuscript. Their study included two groups $(N=50)$ : bilingual Puerto Rican immigrants who immigrated voluntarily to the USA from the island between the ages of 22-28 $(S D=5.61)$ with mean age $69.68(S D=7.69)$, and monolingual Puerto Rican citizens who remained on the island, with mean age of $72.09(S D=5.35)$. The researchers cued the bilingual participants in separate, language-specific sessions. Participants rated the intensity of each memory with a 5-point Likert scale: "I can feel now what I felt then, 1 (not at all) to 5 (as clearly as if it were happening right now). Next, the researchers coded each event's event valence with a 5-point Likert scale, 1 (very negative) to 5 (very positive). Despite the many differences between this study and the data presented in the present study, the results were similar. Schrauf and Hoffman found evidence of affective fading such that older memories were associated with less affect intensity than recent memories. Consistent with the research on the FAB, this temporal difference was greater for negative events than for positive events.

\section{General Discussion}

The FAB occurred in each of the samples that we examined, regardless of the cultural background of the participants. Such findings suggest that the FAB is likely not limited to the U.S.; indeed, it occurred for those who were outside of the U.S. In terms of effect sizes, the FAB was stronger in some samples (Native American, Ghanaian, German) and weaker in other 
samples (Irish and Caucasian British); nonetheless, a valence effect was present. From a metaanalytic perspective, most of the ten samples evinced a FAB effect that was well above zero (only one was near zero, under statistically controlled conditions). In fact, half of the samples evidenced a consistent pattern: about a third of the variance in affect fading was accounted for by valence (i.e., African-American, Ghanaian, Caucasian American, Native American, German, and to a slightly lesser extent, Caucasian New Zealander). Most of the individuals in these samples were not from U.S. cultures. Based on our initial findings the FAB is most likely not a cultural phenomenon that is limited to the U.S. Combining these results with the conceptual replication of a FAB in the Puerto Rican samples (Schrauf \& Hoffman, 2007), the implication is clear: The FAB is likely a pancultural phenomenon. We think that the FAB facilitates individuals from diverse backgrounds to process negativity, adapt to changing circumstances, and to retain a positive emotional tone, in tandem with their own local norms and customs.

\section{Context and Method Considerations}

Our findings also suggest that the FAB is not limited to young adults, such as college students. The samples in the present manuscript were diverse in two other important ways: Several samples included non-students and several samples included participants who were well older than age 30. The German, Ghanaian, Native American, and Puerto Rican samples included non-students; the Irish and Caucasian British samples included data collected from the community. Including the participant age variable into the statistical model resulted in a statistically significant FAB. These individual differences contribute to diversity among our data; indeed, too much psychological research has historically focused only on college students who were under the age of 25 (Henrich, Heine, \& Norenzayan, 2010). We currently prepare a 
manuscript that examines the variance in the FAB across the lifespan, from the early teen years through late adulthood (over 90 years old).

The autobiographical events and their ratings reported here all came from retrospective reports of event memories, some of which were a day old, many were more than a decade old (Bohn \& Berntsen, 2007; Schrauf \& Hoffman, 2007). Such reports are subject to retrospective biases that sometimes distort the perceived changes in memory. Greenwald (1980) famously observed that individuals often grossly distort their personal histories to maintain what he referred to as the 'totalitarian ego'. Does the FAB reflect a distortion of memory that makes autobiographical memories more positive? We think so; however, our data do not reflect the gross distortion of reality to which Greenwald referred. We elucidate some of these reasons below.

First, the FAB has been documented by numerous researchers, and via numerous methodologies, which have tested many parameters of the phenomenon (Gibbons et al., 2011; Holmes, 1970; Ritchie et al., 2006, 2009; Walker et al., 1997). Regardless of design or measurement nuances, the data clearly identified the source of the FAB to be in ratings of current affect, not the ratings of initial affect (Landau \& Gunter, 2009). Importantly, the extant data in support of the FAB suggest that participants did not re-appraise their past events to create alternate versions of the past; rather, they reinterpreted their events in light of their current circumstances (Skowronski et al., 2004; Ritchie \& Batteson, 2013; Walker et al, 2003b; Walker et al, 2009). We encourage researchers to employ different research methods to study the FAB; however, while sampling across different cultures it would be prudent to maintain methodological consistency. 
Second, this phenomenon has been meaningfully linked to other psychological phenomena, such as current mood (Ritchie, 2006), different types of event rehearsal (Ritchie et al., 2006; Walker et al., 2009), social discourse (Skowronski et al., 2004), flashbulb memory (Bohn \& Berntsen, 2007), psychological closure (Beike \& Crone, 2008; Ritchie et al., 2006), event imagery (Ritchie \& Batteson, 2013), and dreaming (Ritchie \& Skowronski, 2008).

Third, the FAB occurred across levels of individual differences, such as personal theories about emotion change and dispositional mood pleasantness (Ritchie et al., 2009). Evidence suggests that dysphoria, anxiety, alcohol consumption, and recreational substance usage disrupt the FAB (Gibbons et al., 2013; Ritchie \& Skowronski, 2008; Walker, et al., 2003a; Walker, Yancu, Skowronski, \& Vogl, 2013), and for those who recalled events of regret and disappointment (Beike \& Crone, 2008).

In sum, to date, even though the FAB has been studied primarily within the U.S. using a relatively wide variety of research methods, a consistent pattern emerged. In the present study, we adopted a similar multi-method approach to the study of the FAB across U.S. regions and in other countries. Consistent with the extant FAB studies, we think that the limitations of one kind of research method were made up for by the strengths of another method. Most importantly, the converging results from different methods reinforce the idea that the FAB is not an artifact of a particular research method. Crucially, despite the different methods used in the present study, the FAB occurred both within and outside of the U.S.

\section{Conceptual Considerations}

Initially, we interpreted the FAB in terms of Taylor's (1991) mobilization-minimization hypothesis. According to this hypothesis, emotional events invoke strong responses in a person's 
physiological, cognitive, and social systems. This initial response represents the mobilization of resources to deal with the immediate consequences of a negative event. For instance, imagine failing a test. When learning of a failing grade, a person's body might respond by releasing stress hormones (i.e., dry mouth, increased blood pressure). Attention may narrow to focus on specific aspects of the event (i.e., specific information that lead to failure), in part, to help the person attempt to understand what went awry. In turn, the person might call upon a friend or family member to help them cope with the failure, such as to calm the person and to plan a study session before the next test. Sometime after the negative event, the person minimizes the long-term adverse effects of the event through a set of opponent processes. The nervous system manages the stress hormones (i.e., maintains basal levels), remains relaxed and attentive, and the person pursues goals for test preparation. According to this hypothesis, mobilization and minimization are almost always stronger for negative events than for positive events. The combination of mobilization and minimization is beneficial in at least two ways.

First, the combination allows individuals to buffer the effects of negative events. In order to maintain a generally positive outlook, people need to be able to reevaluate negative experiences in the larger context of the life experience. Indeed, failing a test can seriously hurt a grade or even career prospects; however, many individuals have endured far worse events and dealt with such events successfully. We think that the FAB plays a significant role in acts of resilience. Further, mobilization and minimization allows individuals the opportunity to soften the impact that negative events often have, if not in the short-term, then most certainly in the long-term. 
Second, positive events evoke a much different response. The aftermath of a positive event does not call for resource minimization. Without a crisis to manage, the person draws upon comparatively fewer resources. Positive events tend to offer opportunities to broaden and build a person’s psychological resources. The Broaden and Build Theory of Positive Emotions (Fredrickson, 2001), proposes that positive emotions and events promote behaviors associated with play, exploration, and creativity. Some of these behaviors occur in private, however many positive emotions promote the formation of, and strengthen existing, interpersonal relationships. Positive emotions can also minimize the intensity of negative emotions, in part, by putting those emotions in a broader perspective. Because of their broadening effect, positive emotions increase the probability of seeking out and finding positive events in the future. Maintaining the affect associated with positive life events can help to foster these emotions and the behaviors that they engender.

The results of the present study also converge with Keltner and Haidt’s (1999; Keltner et al., 2006) conceptualization of the social functions of emotion. This model posited that emotions have universal functions that exist at the levels of the individual and in social interactions. The data suggest that the FAB is a universal phenomenon, so far, with relatively minimal cultural variation. We argue that the FAB is part of multiple processes that comprise affect regulation. We suggest that the FAB enables individuals the ability to recover from negative experiences that can range from minor setbacks to national tragedies.

\section{Limitations and Future Research}

While our research covered a lot of ground culturally, and produced converging evidence in support of the FAB, our studies were not perfectly consistent. Purposive sampling is 
inherently more restrictive than convenience sampling; hence, some of our person sample sizes could have benefitted from more participants. Also, we wonder about the extent to which language translation issues influenced our results. That is, one inclusion criterion was that our participants spoke English and could respond to materials that were written in English. Future studies of the FAB should attempt to find evidence of the FAB from event descriptions expressed in the participants' native languages. Indeed, we and others have begun such an endeavor.

To begin to fill this void, Skowronski, Sedikides, Xie, and Zhou (2013) examined memorial positivity biases in multiple Chinese samples, and found corroborating evidence that the FAB occurred. Further, a forthcoming manuscript makes up for the fact that none of our samples represented the FAB among Asian countries and cultures. We have begun to research the FAB among samples from China, Japan, the Philippines, and Kyrgyzstan. Taken together, such findings will indicate the extent to which the $\mathrm{FAB}$ is a pancultural phenomenon. Additionally, in an attempt to evince any developmental issues that might contribute to the FAB, we prepare a multi-study manuscript about the extent to which the FAB depends on a person's age, spanning from early adolescence through late adulthood. We encourage researchers to examine these and other individual differences, in an attempt to clarify the parameters and mechanisms of the FAB.

Further, we caution readers to be mindful of the fact that the FAB is a valence effect rooted in a person's appraisal of their own past events, and how strongly such events provoke feelings in the present. The pattern of means that we report reflect this valence effect and the FAB appears to occur in culturally different U.S. regions and outside of the U.S. We do not 
intend to imply that the FAB occurs for the same reasons around the world. While the FAB could be a set of memorial processes that facilitate emotion regulation, our data do not confirm this definitively. Future research is needed to clarify why the FAB occurs, generally, and the functions that the FAB serves from culture to culture.

\section{Coda}

The Fading Affect Bias represents a fundamental characteristic of the autobiographical memory. The present findings suggest that the FAB occurs across a variety of U.S. regions, and importantly elsewhere in the world. Across cultures, the negative affect associated with life events fades faster than the positive affect associated with such events. We believe that this phenomenon is part of a set of cognitive processes that foster emotion regulation and enable psychological resilience (Diener \& Diener, 1996; Diener, et al., 2010; Lykken \& Tellegen, 1996). Future research is needed to test the FAB in Asia, the Middle East, elsewhere in Africa and South America. We believe that there is much to learn from elucidating the general and local functions of FAB. 


\section{References}

American Psychological Association. (2010). Ethical principles of psychologists and code of conduct. Retrieved January 7, 2014, from http://www.apa.org/ethics/code/index.aspx

Barrett, L. F., Niedenthal, P., \& Winkielman, P. (Eds.) (2005). Emotions: Conscious and Unconscious. New York, NY: Guilford.

Beike, D. R., \& Crone, T. S. (2008). When experienced regret refuses to fade: Regrets of action and attempting to forget open life regrets. Journal of Experimental Social Psychology, 44, 1545-1550. doi: 10.1016/j.jesp.2008.06.008

Bohn, A., \& Berntsen, D. (2007). Pleasantness bias in flashbulb memories: Positive and negative flashbulb memories of the fall of the Berlin wall. Memory and Cognition, 35, 565-577. doi: 10.3758/BF03193295

British Psychological Society. (2009). Code of ethics and conduct: Guidance published by the Ethics Committee of the British Psychological Society. Retrieved January 7, 2014, from http://www.bps.org.uk/sites/default/files/documents/code_of_ethics_and_conduct.pdf

Bryant, F. B., \& Veroff, J. (2007). Savoring: A new model of positive experience.

Mahwah, NJ: Lawrence Erlbaum Associates.

Cason, H. (1932). The learning and retention of pleasant and unpleasant activities. Archives of Psychology, 134, 1-96.

Charles, S. T., \& Carstensen, L. L. (2010). Social and emotional aging. Annual Review of Psychology, 61, 383-409. doi: 10.1146/annurev.psych.093008 
Clark, R., Anderson, N. B., Clark, V. R., \& Williams, D. R. (1999). Racism as a stressor for African-Americans: A biopsychosocial model. American Psychologist, 54 (10), 805-816. doi:10.1037/0003-066X.54.10.805

Damasio, A. (2003). Looking for Spinoza: Joy, sorrow, and the feeling brain, New York, NY: Harcourt.

De Leersnyder, J., Boiger, M., \& Mesquita, B. (2013). Cultural regulation of emotion: Individual, relational, and structural sources. Frontiers in Psychology, 4, 1-11. doi: 10.3389/fpsyg.2013.00055

Diener, E., \& Diener, C. (1996). Most people are happy. Psychological Science, 7, 181-185. doi: 10.1111/j.1467-9280.1996.tb00354.x

Diener, E., Ng, W., Harter, J., \& Arora, R. (2010). Wealth and happiness across the world: Material prosperity predicts life evaluation, whereas psychosocial prosperity predicts positive feeling. Journal of Personality and Social Psychology, 99(1), 52-61. doi: $10.1037 / \mathrm{a} 0018066$.

Dorgan, B. L. (2010). The tragedy of Native-American youth suicide. Psychological Services, 7, (3), 213-218. doi: 10.1037/a0020461

Ekman, P. (1972). Universals and Cultural Differences in Facial Expression of Emotion. In J. Cole ed. Nebraska Symposium on Motivation. (pp. 207-283). Lincoln, Nebraska: University of Nebraska Press.

Ekman, P., \& Friesen, W. V. (1971). Constants across cultures in the face and emotion. Journal of Personality and Social Psychology, 17, 124-129. doi: 10.1037/h0030377 
Elfenbein, H. A., \& Ambady, N. (2002). On the universality and cultural specificity of emotion recognition: A meta-analysis. Psychological Bulletin, 128, 203-235.

doi: 10.1037/0033-2909.128.2.203

Ehrenreich, B. (2009). Bright-sided: How positive thinking is undermining America. New York, NY: Picador.

Forgas, J. P. (Ed.) (2000). Feeling and Thinking: The Role of Affect in Social Cognition. Cambridge, UK: Cambridge University Press.

Fredrickson, B. L. (2001). The role of positive emotions in positive psychology: The broaden and build theory of positive emotions. American Psychologist, 56 (3): 218-226. doi:10.1037/0003-066X.56.3.218

Gibbons, J. A., Lee, S. A., \& Walker, W. R. (2011). The fading affect bias begins within 12 hours and persists for 3 months. Applied Cognitive Psychology, 25, 663-672. doi: 10.1002/acp.1738

Gibbons, J. A., Toscano, A., Kofron, S., Rothwell, C., Lee, S. A., Ritchie, T. D., \& Walker, W. R. (2013). The fading affect bias across alcohol consumption frequency for alcohol-related and non-alcohol-related events. Consciousness and Cognition, 22, 13401351. doi: 10.1016/j.concog.2013.09.004

Glück, J., \& Bluck, S. (2007). Looking back across the lifespan: A life story account of the reminiscence bump. Memory and Cognition, 35, 1928-1939.

Greenwald, A. G. (1980). The totalitarian ego: Fabrication and revision of personal history. American Psychologist, 35, 603-618. doi: 10.1037/0003-066X.35.7.603 
Henrich, J., Heine, S. J., \& Norenzayan, A. (2010). The weirdest people in the world? Behavioral and Brain Sciences, 33(2-3), 61-83. doi: 10.1017/S0140525X0999152X

Holmes, D. S. (1970). Differential change in affective intensity and the forgetting of unpleasant personal experiences. Journal of Personality and Social Psychology, 15, 234-239.

Keltner, D., \& Haidt, J. (1999). Social functions of emotions at four levels of analysis. Cognition and Emotion, 13, 505-521.

Keltner, D., Haidt, J., \& Shiota, M. N. (2006). Social functionalism and the evolution of emotions. In M. Schaller, J. A. Simpson, \& D. T. Kenrick (Eds.), Evolution and Social Psychology (pp. 115-142). Madison, CT: Psychosocial Press.

La France, M., Hecht, M. A., \& Paluck, E. L. (2003). The contingent smile: A meta-analysis of sex differences in smiling. Psychological Bulletin, 129, 305-334. doi: 10.1037/0033-2909.129.2.305

Landau, J. D., \& Gunter, B. C. (2009). “Don’t worry: you really will get over it”: Methodological investigations of the fading affect bias. American Journal of Psychology, 122, 209-217.

Lykken, D., \& Tellegen, A. (1996). Happiness is a stochastic phenomenon. Psychological Science, 7, 183-189. doi: 10.1111/j.1467-9280.1996.tb00355.x

Matsumoto, D., \& Hwang, H. S. (2010). Judging faces in context. Social and Personality Psychology Compass, 4, 393-402. doi: 10.1111/j.1751-9004.2010.00271.x

McAdams, D. P. (2013). The redemptive self: Stories Americans live by - Revised and expanded edition. New York, NY, US: Oxford University Press. 
Meltzer, H. (1930). Individual differences in forgetting pleasant and unpleasant experiences. Journal of Educational Psychology, 21, 399-409.

Meltzer, H. (1931). Sex differences in forgetting pleasant and unpleasant experiences. Journal of Abnormal and Social Psychology, 25, 450-464.

Quoidbach, J., Berry, E., Hansenne, M., \& Mikolajczak, M. (2010). Positive emotion regulation and well-being: Comparing the impact of eight savoring and dampening strategies. Personality and Individual Differences, 49, 368-373. doi: 10.1016/j.paid.2010.03.048

Ritchie, T. D. (2006). Trait affect, state affect, and the fading affect bias in autobiographical memory. Doctoral dissertation, Northern Illinois University.

Ritchie, T. D., \& Batteson, T. J. (2013). Perceived changes in ordinary autobiographical events’ affect and visual imagery colorfulness. Consciousness and Cognition, 22, 461-470. doi: 10.1016/j.concog.2013.02.001

Ritchie, T. D., \& Dromey, M. (2014). Eating disorder symptoms disrupt the fading affect bias in autobiographical memory. Unpublished manuscript, University of Limerick.

Ritchie, T. D., \& Skowronski, J. J. (2008). Perceived change in the affect associated with dreams: The fading affect bias and its moderators. Dreaming, 18, 27-43. doi: 10.1037/1053-0797.18.1.27

Ritchie, T. D., Skowronski, J. J., Cadogan, S., \& Sedikides, C. (in press). Affective responses to self-defining autobiographical events. Self and Identity. doi:10.1080/15298868.2013.863222 
Ritchie, T. D., Walker, W. R., Marsh, S., \& Skowronski, J. J. (2014). Narcissism distorts the fading affect bias in autobiographical memory. Unpublished manuscript, University of Limerick.

Ritchie, T. D., Skowronski, J. J., Wood, S. E., Walker, W. R., Vogl, R., \& Gibbons, J. (2006). Event self-importance, event rehearsal, and the fading affect bias in autobiographical memory. Self and Identity, 5, 172-195. doi: 10.1080/15298860600591222

Ritchie, T. D., Skowronski, J. J., Hartnett, J. L., Wells, B. M., \& Walker, W. R. (2009). The fading affect bias in the context of emotion activation level, mood, and personal theories of emotion change. Memory, 17, 428-444. doi: 10.1080/09658210902791665

Scherer, K. R., \& Brosch, T. (2009). Culture-specific appraisal biases contributed to emotion dispositions. European Journal of Personality, Special Issue: Personality and Culture, 23(3), 265-288. doi: 10.1002/per.714

Schrauf, R.W., \& Hoffman, L. (2007). The effects of revisionism on remembered emotion: The valence of older, voluntary immigrants’ pre-migration autobiographical memories. Applied Cognitive Psychology, 21, 895-913. doi: 10.1002/acp.1304

Skowronski, J. J., Gibbons, J. A., Vogl, R. J., \& Walker, W. R. (2004). The effect of social disclosure on the intensity of affect provoked by autobiographical memories. Self and Identity, 3, 285-309. doi: 10.1080/13576500444000065

Taylor, S. E. (1991). Asymmetrical effects of positive and negative events: The mobilizationminimization hypothesis. Psychological Bulletin, 110, 67-85. doi: 10.1037/0033-2909.110.1.67

Twenge, J. M., \& Campbell, W. K. (2009). The narcissism epidemic. New York, NY: Atria 
books.

Walker, W. R. (1998). Dysphoria and autobiographical memory: How pleasant and unpleasant memories are remembered. Doctoral dissertation, Kansas State University.

Walker, W. R., \& Skowronski, J. J. (2009). The Fading Affect Bias: But What the Hell is It For? Applied Cognitive Psychology, 23, 1122-1136. doi: 10.1002/acp.1614

Walker, W. R., Skowronski, J. J., \& Thompson, C. P. (2003b). Life is pleasant - and memory helps to keep it that way! Review of General Psychology, 7, 203-210. doi: 10.1037/1089-2680.7.2.203

Walker, W. R., Vogl, R. J., \& Thompson, C. P. (1997). Autobiographical memory: Unpleasantness fades faster than pleasantness over time. Applied Cognitive Psychology, 11, 399-413. doi: 10.1002/(SICI)1099-0720(199710)11:5<399::AID-ACP462>3.0.CO;2-E

Walker, W. R., Yancu, C. N., Skowronski, J. J., \& Vogl, R. J. (2014). Dispositional anxiety reduces affective fading for positive and negative events. Unpublished manuscript, Winston-Salem State University.

Walker, W. R., Skowronski, J. J., Gibbons, J. A., Vogl, R. J., \& Ritchie, T. D. (2009). Why people rehearse their memories: Frequency of use and relations to the intensity of emotions associated with autobiographical memories. Memory, 17, 760-773. doi: 10.1080/09658210903107846

Walker, W. R., Skowronski, J. J., Gibbons, J. A., Vogl, R., \& Thompson, C. P. (2003a). On the emotions that accompany autobiographical memories: Dysphoria disrupts the fading affect bias. Cognition and Emotion, 17, 703-723. doi: 10.1080/02699930302287 
This is an Author's Accepted Manuscript of an article published in Memory, epub ahead of print published online:14th February 2014, available online at: http://www.tandfonline.com/10.1080/09658211.2014.884138

Watters, R., \& Leeper, R. (1936). The relation of affective tone to the retention of experiences in everyday life. Journal of Experimental Psychology, 19, 203-215. 
Table 1

Person and Event Sizes per Sample

\begin{tabular}{ccccc}
\hline Sample & Location & Ethnic Identity & $N_{\text {Participants }}$ & $N_{\text {Events }}$ \\
\hline 1 & & USA & 58 & 572 \\
\cline { 2 - 5 } 2 & Africa & Ghanaian & 14 & 121 \\
3 & USA & Caucasian American & 30 & 186 \\
\cline { 2 - 5 } 4 & USA & Native American & 25 & 85 \\
5 & Germany & Caucasian German & 180 & 180 \\
\cline { 2 - 5 } 6 & New Zealand & European ancestry & 43 & 429 \\
7 & New Zealand & Maori \& Pasifica ancestry & 16 & 161 \\
\cline { 2 - 5 } 8 & UK & Caucasian British & 61 & 244 \\
9 & Ireland & Caucasian Irish & 100 & 395 \\
\cline { 2 - 5 } 10 & UK & Black British & 33 & 66 \\
\cline { 2 - 5 } Total & & & 562 & 2439 \\
\cline { 2 - 5 } & & & & \\
\cline { 2 - 5 }
\end{tabular}


Table 2

Absolute Values of the Raw Means and Standard Errors for Ratings of Initial Affect, Current Affect, and Affect Fading per Sample

\begin{tabular}{ccccccc}
\hline & \multicolumn{2}{c}{ Negative Affect } & Positive Affect & Faded Affect \\
\cline { 2 - 6 } Sample & Initial & Current & Initial & Current & Negative & Positive \\
& & & & & & \\
\hline African-American & $2.25(.07)$ & $0.98(.09)$ & $2.51(.07)$ & $1.81(.09)$ & $1.27(.09)$ & $0.70(.09)$ \\
\hline Ghanaian & $2.58(.12)$ & $1.02(.19)$ & $2.68(.12)$ & $2.04(.19)$ & $1.56(.20)$ & $0.64(.20)$ \\
\hline Caucasian American & $2.32(.10)$ & $1.00(.14)$ & $2.37(.09)$ & $2.00(.12)$ & $1.32(.12)$ & $0.37(.11)$ \\
\hline Native American & $1.93(.14)$ & $0.78(.18)$ & $2.23(.14)$ & $2.16(.18)$ & $1.15(.14)$ & $0.07(.14)$ \\
\hline German & $2.28(.09)$ & $1.10(.04)$ & $2.48(.07)$ & $1.97(.13)$ & $1.18(.15)$ & $0.50(.11)$ \\
\hline Caucasian New Zealander & $2.42(.06)$ & $1.47(.07)$ & $2.41(.06)$ & $2.01(.07)$ & $0.96(.07)$ & $0.40(.07)$ \\
\hline Maori \& Pasifica & $2.15(.15)$ & $1.43(.18)$ & $2.36(.15)$ & $1.99(.12)$ & $0.73(.15)$ & $0.37(.14)$ \\
\hline Caucasian British & $5.09(.11)$ & $3.54(.15)$ & $5.34(.10)$ & $4.82(.14)$ & $1.56(.13)$ & $0.52(.13)$ \\
\hline Irish ${ }^{\mathrm{a}}$ & & & & & & \\
\hline & $5.14(.09)$ & $3.02(.11)$ & $5.28(.09)$ & $4.73(.11)$ & $2.12(.11)$ & $0.56(.11)$ \\
\hline & & & & & & \\
\hline
\end{tabular}

Notes. ${ }^{\text {a}}$ Fading Affect valence effect rendered null when individual differences in event recall was included in the model. 
Table 3

Variance Explained in Affect Fading by Individual Differences (Person), Relative to Variance Explained in Affect Fading by Person and Event Valence $\left(\Delta R^{2}\right)$

\begin{tabular}{|c|c|c|c|}
\hline & \multicolumn{3}{|c|}{ Variance Explained in Affect Fading } \\
\hline & Person & Person + Event Valence & Effect Size Change \\
\hline Sample & $R^{2}$ & $R^{2}$ & $\Delta R^{2}$ \\
\hline African-American & .18 & .49 & .31 \\
\hline Ghanaian & .11 & .44 & .33 \\
\hline Caucasian American & .22 & .52 & .30 \\
\hline Native American & .27 & .61 & .34 \\
\hline Caucasian British & .27 & .40 & .13 \\
\hline German $^{\mathrm{a}}$ & -- & .32 & -- \\
\hline Caucasian New Zealander & .10 & .38 & .28 \\
\hline Maori \& Pasifica & .12 & .28 & .16 \\
\hline Irish $^{\mathrm{b}}$ & .52 & .53 & .01 \\
\hline Black British $^{\mathrm{c}}$ & .77 & .93 & .16 \\
\hline Votes. ${ }^{\mathrm{a}}$ Data were not clustere & (1) & mples. ${ }^{b}$ Valence effect & on-significant whe \\
\hline
\end{tabular}

